\title{
Symmetries of the Trautman retarded radial coordinates
}

\author{
Maciej Kolanowski* and Jerzy Lewandowskit \\ Institute of Theoretical Physics, Faculty of Physics, \\ University of Warsaw, Pasteura 5, 02-093 Warsaw, Poland
}

\begin{abstract}
We consider spacetime described by observer that uses Trautman's retarded radial coordinates system. Given a metric tensor, we find all the symmetry maps. They set a 10 dimensional family of local diffeomorphisms of spacetime and can be parametrized by the Poincaré algebra. This result is similar to the symmetries of a Gauss observer using the Gauss simultaneous radial coordinates and experiencing the algebra deformation induced by the spacetime Riemann tensor. A new, surprising property of the retarded coordinates is that while the symmetries are differentiable, in general they are not differentiable twice. In other words, a family of smooth symmetries is smaller than in the Gaussian case. We demonstrate examples of that non-smoothness and find necessary conditions for the differentiability to the second order. We also discuss the consequences and relevance of that result for geometric relational observables program. One can interpret our result as that the retarded radial coordinates provide gauge conditions stronger that the Gaussian 4d radial gauge.
\end{abstract}

PACS numbers: 04.70.Bw, 04.50.Gh

* mp.kolanowski@student.uw.edu.pl

$\dagger$ Jerzy.Lewandowski@fuw.edu.pl 


\section{INTRODUCTION}

In general relativity we often use coordinates adapted in a natural way to a given metric tensor. In that way we reduce the degrees of freedom in the metric tensor components related to non-physical and non-geometrical ambiguities. Therefore one may thing of that choice of coordinates as a gauge fixing. The diffeomorphisms that destroy our gauge get eliminated. Usually, though, there still remains a family of diffeomorphisms that preserve the conditions imposed on the coordinates. They are symmetries of a framework based on that gauge choice. An example of adapted coordinates may be Trautman - Bondi coordinates defined in asymptotically flat spacetime in a neighborhood of conformally defined boundary of spacetime [1, 2]. Their symmetries set the famous Bondi-Misner-Sachs (BMS) group. It is infinite dimensional, contains infinitely many subgroups isomorphic to the Poincaré group. The relevance of the BMS group for generally relativistic physics is very well known. In the current paper we consider coordinates adapted in a similar way to a timelike geodesic curve - an observer. We use incoming null geodesic curves, proper time, affine parametrizations, and angles parametrizing the sphere of null directions parallely transported along the observer's world line. Such coordinates were introduced for the first time by Trautman [3] in his search for special solutions to Einstein's equations, therefore we will name them after him. Our work arises from the program of construction of diffeomorphism invariant observables for canonical general relativity naturally related to geometry [4]. This is a geometric version of the idea of the relational observables [5]. In the previous papers we considered in that context radial coordinates in 3d Euclidean space [6], and Gauss radial coordinates in 4d spacetime, respectively [7]. Similar construction was considered for asymptotically AdS spacetimes [8]. Analysis of (non-)locality of such observables was performed in perturbative approach [9]. We found the symmetries of said coordinate systems and analyzed their structure [10]. In the case of the radial coordinates in the Euclidean 3d space, for every a metric tensor, the symmetries set a 6 dimensional family that may be parametrized by the Lie algebra of the isometries of Euclidean flat space. In the case of the Gauss radial coordinates in $4 \mathrm{~d}$ spacetime, observer's symmetries form a 10 dimensional family parametrized by the Poincaré algebra. In the both cases, though, the symmetries are not a group. Composition of two symmetries of a given metric tensor may happen not to be a symmetry any more. On the other hand, they do induce a group of motions of the space of the metric tensors. The generators of that group satisfy the commutation relations of the parameterizing Lie algebra suitably deformed by the Riemann curvature tensor (translations do not commute). The Trautman coordinates are more physical because of their retarded character. They are most difficult, though, from the point of view of the symplectic structure of Einstein's theory of gravity and other fields. In the current paper we investigate symmetries of the Trautman coordinates. A priori, one would expect that they again form a 10 dimensional family per a metric tensor parametrized by the Poincaré algebra. We find that this guess is partially true with a catch, that the symmetries fail to be twice differentiable. We provide examples, find some 
second differentiability conditions and discuss the relevance of that surprising result.

\section{TRAUTMAN'S RETARDED RADIAL COORDINATES}

We consider a 4-dimensional spacetime $M$ and metric tensor $g$ of the signature -+++ . Observer world line is a time-like curve in $M$

$$
\mathbb{R} \ni \tau \mapsto p(\tau) \in M
$$

where $\tau$ is the proper time, that is

$$
\frac{d p^{\mu}}{d \tau} \frac{d p_{\mu}}{d \tau}=-1
$$

We are assuming throughout this paper that the observer world line is geodesic

$$
\nabla_{\frac{d p}{d \tau}} \frac{d p}{d \tau}=0
$$

Our considerations are local, for example we do not bring up the issue of extendability of the observer line.

Trautman coordinates are defined in a neighborhood of the observer world line. For each point $p(\tau)$ of the observer world line we consider the past null cone formed by incoming null geodesics (generators). On each null generator of the cone we introduce an affine coordinate $r$ growing to the past. Let $n^{\mu}$ be the corresponding null vector tangent to the cone and normalized such that

$$
n^{\mu} \partial_{\mu} r=1
$$

The ambiguity in the choice of the $r$ coordinate is fixed by assuming the following two conditions:

$$
r=0
$$

at the end of each generator (that is the intersection of the generator with the observer world line), and

$$
n^{\mu} \frac{d p_{\mu}}{d \tau}=1
$$

Each null geodesic curve coming in a point at the observer world line can be characterized by the intersection point and two angle variables $\theta^{A}=\theta, \phi$ parameterizing the sphere of null directions at that point. We assume that the angles $\theta^{A}$ are constant with respect to the parallel transport of the null vectors along the observer world line. Now, to every point $m$ in a sufficiently narrow neighborhood of the observer world line (and not contained 
in the world line itself) there corresponds uniquely: a null curve connecting $m$ with the observer world line, the value $\tau(m)$ of the observer time at the intersection point, the value $r(m)$ of the affine parameter corresponding to the point $m$, and the angles $\theta(m), \phi(m)$ characterizing the null geodesic curve. In that way we have introduced a coordinate system $(\tau, r, \theta, \phi)$

$$
m \mapsto(\tau(m), r(m), \theta(m), \phi(m))
$$

in the neighborhood of the observer world line that is singular at the world line. We call that neighborhood the domain of the coordinates (despite the singularity at $r=0$ ). In those coordinates the metric tensor components satisfy a very simple (gauge) condition

$$
g_{r \mu}=\delta_{\tau \mu}
$$

We have to remember, however, about additional very non-trivial (gauge) conditions implied by the above construction and by the differentiability of the metric tensor $g$ - for simplicity let us assume $g$ is smooth, but in fact we will use only the second derivatives of $g$ to calculate it's Riemann tensor - on the components $g_{\tau \tau}, g_{\tau A}, g_{A B}$ and their derivatives to arbitrary order in the limit as $r \mapsto 0$.

Given a metric tensor $g$, each system of Trautman coordinates is determined by a point $m_{0} \in M$ and an orthonormal tangent frame $e_{0}, e_{1}, e_{2}, e_{3} \in T_{m_{0}} M$. Indeed, the observer world line is determined by the time like vector $e_{0}$, the proper time $\tau$ is chosen to vanish at $m_{0}$, the vectors $e_{1}, e_{2}, e_{3}$ determine in a standard way a spherical coordinates system $\theta, \phi$ on the sphere of the null directions at $m_{0}$. This correspondence

$$
(\tau, r, \theta, \phi) \leftrightarrow\left(m_{0}, e_{0}, e_{1}, e_{2}, e_{3}\right)
$$

is 1 -to- 1 .

\section{SYMMETRIES}

Given a metric tensor $g$ and Trautman coordinates $(\tau, r, \theta, \phi)$, a symmetry is a local diffeomorphism $f$ defined in an open and connected region contained in the domain of the coordinates and containing a segment of the observer line, such that for the pulled back metric tensor $f^{*} g$ the functions $(\tau, r, \theta, \phi)$ also satisfy the definition of Trautman coordinates. In other words, $f$ is a symmetry whenever the metric tensor $f^{*} g$ also satisfies the gauge conditions (8) and the conditions described below (8).

We find now all the symmetries modulo a catch we will point out at the end.

Suppose $f$ is a symmetry. Then, the functions $f^{-1^{*}} \tau, f^{-1^{*}} r, f^{-1^{*}} \theta, f^{-1^{*}} \phi$ set another system of retarded radial coordinates for the metric tensor $g$. Therefore, every symmetry of a given Trautman coordinates $(\tau, r, \theta, \phi)$ can be obtained in the following way: let 
$\left(\tau^{\prime}, r^{\prime}, \theta^{\prime}, \phi^{\prime}\right)$ be another system of Trautman coordinates for the metric tensor $g$. Define $f$ by the equation

$$
(\tau(m), r(m), \theta(m), \phi(m))=\left(\tau^{\prime}(f(m)), r^{\prime}(f(m)), \theta^{\prime}(f(m)), \phi^{\prime}(f(m))\right)
$$

Conversely, every map $f$ defined in that way is a symmetry of $g$ and $(\tau, r, \theta, \phi)$.

Now, the catch is the differentiability level of the map $f$ at the points of the observer world line. Indeed, at the points of $M$ such that $r>0\left(r^{\prime}>0\right)$, the coordinates $(\tau, r, \theta, \phi)$ $\left(\left(\tau^{\prime}, r^{\prime}, \theta^{\prime}, \phi^{\prime}\right)\right)$, and in the consequence $f$, are all smooth. At the world line, on the other hand, our Trautman coordinates are not very useful to judge about the differentiability. However, at a point $p\left(\tau_{1}\right)$ on the observer world line (11), every vector $X \in T_{p\left(\tau_{1}\right)} M$ can be uniquely written as

$$
X=a \frac{d p}{d \tau}\left(\tau_{1}\right)+b \partial_{r}^{\left(\theta_{1}, \phi_{1}\right)}, \quad a, b \in \mathbb{R}
$$

where $\partial_{r}^{\left(\theta_{1}, \phi_{1}\right)}$ is the limit $r \mapsto 0$ of the vector field $\partial_{r}$ along a null geodesic curve corresponding to $\left(\tau_{1}, \theta_{1}, \phi_{1}\right)$. Every curve tangent to $X$ is mapped by the symmetry $f$ to a curve tangent to a vector

$$
X^{\prime}=a \frac{d f(p)}{d \tau^{\prime}}\left(\tau_{1}\right)+b \partial_{r^{\prime}}^{\left(\theta_{1}, \phi_{1}\right)}
$$

It is easy to see that this map

$$
T_{p\left(\tau_{1}\right)} M \ni a \frac{d p}{d \tau}\left(\tau_{1}\right)+b \partial_{r}^{\left(\theta_{1}, \phi_{1}\right)} \mapsto a \frac{d f(p)}{d \tau^{\prime}}\left(\tau_{1}\right)+b \partial_{r^{\prime}}^{\left(\theta_{1}, \phi_{1}\right)} \in T_{f(p)\left(\tau_{1}\right)} M
$$

is linear and actually it is the derivative $f_{*}$ of the map $f$ at $p\left(\tau_{1}\right)$.

In summary, given a metric tensor $g$ and Trautman coordinates $(\tau, r, \theta, \phi)$, we have defined and characterized the 10 dimensional family of the symmetries. Obviously, the symmetries depend on the coordinates $(\tau, r, \theta, \phi)$. What they essentially depend on is the underlying observer world line $p$, modulo the beginning point $p(0)$. Therefore we can denote them $\operatorname{Sym}(g, p)$. Notice, that if $g \neq g^{\prime}$ then in general

$$
\operatorname{Sym}\left(g^{\prime}, p\right) \neq \operatorname{Sym}(g, p) \text {. }
$$

For that reason, $\operatorname{Sym}(g, p)$ is not a group except when $g$ is Minkowski, de Sitter, or anti-de Sitter. The symmetries are everywhere differentiable at least once. In general though, a symmetry of Trautman coordinates is not twice differentiable at the observer line (see below). 


\section{EXAMPLE OF A NON-C ${ }^{2}$ SYMMETRY}

In this section we give an example of a metric tensor $\tilde{g}$, Trautman coordinates $(\tilde{\tau}, \tilde{r}, \tilde{\theta}, \tilde{\phi})$ and a symmetry $\tilde{f}$ defined by another Trautman coordinates system $\left(\tilde{\tau}^{\prime}, \tilde{r}^{\prime}, \tilde{\theta}^{\prime}, \tilde{\phi}^{\prime}\right)$ that is not twice differentiable at points on the observer world line.

The construction of our example is suggested by the following observation. Given a metric tensor $g$ and Trautman coordinates $(\tau, r, \theta, \phi)$, one may introduce Cartesian coordinates $(T, X, Y, Z)=\left(T, X^{i}\right)$ defined as follows

$$
\begin{aligned}
T & =\tau-r \\
X & =r \sin \theta \cos \phi \\
Y & =r \sin \theta \sin \phi \\
Z & =r \cos \theta
\end{aligned}
$$

In terms of them, the formula (10) for a symmetry $f$ takes an analogous form

$$
(T(m), X(m), Y(m), Z(m))=\left(T^{\prime}(f(m)), X^{\prime}(f(m)), Y^{\prime}(f(m)), Z^{\prime}(f(m))\right)
$$

where $\left(T^{\prime}, X^{\prime}, Y^{\prime}, Z^{\prime}\right)$ are the Cartesian coordinates corresponding to the primed Trautman coordinates. Now, suppose, that the metric tensor $g$ is flat in a neighborhood of the primed observer's world line, contained in the domain of the primed coordinates $\left(\tau^{\prime}, r^{\prime}, \theta^{\prime}, \phi^{\prime}\right)$. Then, the primed Cartesian coordinates $\left(T^{\prime}, X^{\prime}, Y^{\prime}, Z^{\prime}\right)$ are smooth including the primed world line $r^{\prime}=0$. Obviously, in terms of them the metric tensor is just

$$
g=-d T^{\prime 2}+d X^{\prime 2}+d Y^{\prime 2}+d Z^{\prime 2}
$$

in the flatness region. It follows from (16), that at every point $m$, the symmetry $f$ is exactly as many times differentiable as the map

$$
m \mapsto(T(m), X(m), Y(m), Z(m)) .
$$

Therefore, to construct an example of at most once differentiable symmetry it is sufficient to find a metric tensor $g$ and retarded radial coordinates $(\tau, r, \theta, \phi)$ such that the corresponding Cartesian functions are at most once differentiable. Then, the example will be given by gluing a neighborhood of observer's world line of the Trautman coordinates $(\tau, r, \theta, \phi)$ endowed with the metric $g$, with a neighborhood of observer's world line of the Trautman coordinates $\left(\tau^{\prime}, r^{\prime}, \theta^{\prime}, \phi^{\prime}\right)$ endowed with the flat metric $g^{\prime}$. The gluing is such that the neighborhoods become disjoint neighborhoods in a single spacetime $M$, and a metric $\tilde{g}$ is such that in one neighborhood $\tilde{g}=g$ and in the other one $g=g^{\prime}$. The corresponding symmetry $\tilde{f}=f$ given by (10).

We give now an example of a metric $g$ and Trautman coordinates $(\tau, r, \theta, \phi)$ such that the corresponding Cartesian coordinates are not differentiable more than once. 
Let us consider FLRW spacetime filled with radiation. In the standard cartesian coordinates $(t, x, y, z)$ the metric takes following form

$$
g=-d t^{2}+t\left(d x^{2}+d y^{2}+d z^{2}\right)
$$

One can easily integrate the null geodesic equation. Without loss of generality consider it in the $y=0=z$ plane. We have following equations

$$
\left\{\begin{array}{l}
\left(\frac{d t}{d r}\right)^{2}=t\left(\frac{d x}{d r}\right)^{2} \\
t \frac{d r}{d \lambda}=\text { const. }=\sqrt{\tau}
\end{array}\right.
$$

where $r$ is an affine parameter, the second line comes from properties of Killing vector $\partial_{x}$ and $\tau$ is the time when geodesic crosses observer's word line. By direct integration we obtain

$$
\left\{\begin{array}{l}
t=\tau\left(1-\frac{3 r}{2 \tau}\right)^{\frac{2}{3}} \\
x=-2 \sqrt{\tau}\left(1-\frac{3}{2} \frac{r}{\tau}\right)^{\frac{1}{3}}+2 \sqrt{\tau}
\end{array}\right.
$$

Generalization for any radial coordinate is straightforward. Non-zero elements of metric in those new coordinates $(\tau, r, \theta, \phi)$ are as follows

$$
\left\{\begin{array}{l}
g_{\tau \tau}=\frac{r}{\tau}-\left(1-\frac{3 r}{2 \tau}\right)^{2 / 3}-2 \\
g_{\tau r}=g_{r \tau}=1 \\
g_{\theta \theta}=\tau^{2}\left(1-\frac{3 r}{2 \tau}\right)^{2 / 3}\left(2 \sqrt[3]{1-\frac{3 r}{2 \tau}}-2\right)^{2} \\
g_{\phi \phi}=\tau^{2}\left(1-\frac{3 r}{2 \tau}\right)^{2 / 3}\left(2 \sqrt[3]{1-\frac{3 r}{2 \tau}}-2\right)^{2} \sin ^{2}(\theta)
\end{array}\right.
$$

It is easy to see that the gauge conditions (8) are satisfied. We can also use obtained results to find associated Cartesian coordinates. Firstly, one can invert (21)

$$
\left\{\begin{array}{l}
\tau=\frac{(x+2 \sqrt{t})^{2}}{4} \\
r=\frac{2}{3}\left(\frac{(x+2 \sqrt{t})^{2}}{4}-\frac{2 t \sqrt{t}}{x+2 \sqrt{t}}\right)
\end{array}\right.
$$

which, using (15), gives us

$$
\left\{\begin{array}{l}
T=\tau-r=\frac{\left(\sqrt{x^{2}+y^{2}+z^{2}}+2 \sqrt{t}\right)^{2}}{12}+\frac{4}{3} \frac{t \sqrt{t}}{\sqrt{x^{2}+y^{2}+z^{2}}+2 \sqrt{t}} \\
X^{i}=r \frac{x^{i}}{x^{2}+y^{2}+z^{2}}=\frac{1}{6} x^{i} \frac{x^{2}+y^{2}+z^{2}+6 \sqrt{x^{2}+y^{2}+z^{2}} \sqrt{t}+12 t}{\sqrt{x^{2}+y^{2}+z^{2}}+2 \sqrt{t}}=\sqrt{t} x^{i}+x^{i} \frac{x^{2}+y^{2}+z^{2}}{6 \sqrt{x^{2}+y^{2}+z^{2}}+12 \sqrt{t}}
\end{array}\right.
$$

The non-smoothness at $x=y=z=0$ follows from the presence of square root in denominator. Specifically, each of the functions $T, X, Y, Z$ is everywhere differentiable in 
accordance with the conclusion of the previous section. But consider the mixed second derivative:

$$
\begin{gathered}
\frac{\partial^{2} T}{\partial x \partial y}=\frac{x y \sqrt{t}}{3\left(x^{2}+y^{2}+z^{2}\right)^{\frac{3}{2}}}\left(\frac{4 t}{\left(2 \sqrt{t}+\left(x^{2}+y^{2}+z^{2}\right)^{\frac{1}{2}}\right)^{2}}-1\right)+ \\
\frac{8 t \sqrt{t}}{3\left(2 \sqrt{t}+\sqrt{x^{2}+y^{2}+z^{2}}\right)^{2}} \frac{x y}{\sqrt{x^{2}+y^{2}+z^{2}}}\left(1+\frac{2 \sqrt{t}}{\sqrt{x^{2}+y^{2}+z^{2}}}\right)
\end{gathered}
$$

The very last term in this whole expansion is the only one which is of order $t$ (the rest is of order $\sqrt{t}$ ) and it is clearly non-continuous so our coordinates are not $C^{2}$.

\section{THE SECOND DIFFERENTIABILITY CONDITIONS IN THE FLAT OBSERVER CASE}

In this section we continue the study of the symmetries and their construction via the formulae (10) and (16). As in the example of the previous section, we again assume that the metric $g$ is flat in a neighborhood of the primed observer world line where the primed Cartesian coordinates are used, that is (17) holds there again. However, now we find necessary conditions on $g$ (and its Riemann tensor) that hold at the unprimed observer world line for the existence of the second derivative of the corresponding symmetry $f$ at observer's world line. The condition we derive below reads

$$
\left.R_{\alpha \beta \gamma \delta} \frac{d p^{\delta}}{d \tau}\right|_{p(\tau)}=0
$$

for every point $p(\tau)$ of the world line, where $R^{\alpha}{ }_{\beta \gamma \delta}$ is the Riemann tensor of $g$. We show below the calculation that leads to that conclusion.

If the cartesian coordinates $(T, X, Y, Z)$ (15) are $C^{2}$, then so are $g_{I J}$ as functions of these coordinates. In terms of those coordinates the gauge conditions (8) read as follows

$$
\left\{\begin{array}{l}
-g_{T T}+g_{T X^{i}} \frac{X^{i}}{r}=-1 \\
g_{T T}+g_{i j} \frac{X^{i} X^{j}}{r^{2}}-2 g_{T X^{i}} \frac{X^{i}}{r}=0 \\
\frac{Z}{\rho}\left(-g_{T X} X-g_{T Y} Y\right)+g_{T Z} \rho+\frac{Z}{\rho r}\left(g_{X X} X^{2}+2 g_{X Y} X Y+g_{Y Y} Y^{2}\right)+ \\
\frac{-X^{2}-Y^{2}+Z^{2}}{\rho r}\left(g_{X Z} X+g_{Y Z} Y\right)-g_{Z Z} \frac{Z \rho}{r}=0 \\
g_{T X} Y-g_{T Y} X+\left(g_{Y Y}-g_{X X}\right) \frac{X Y}{r}+g_{X Y} \frac{X^{2}-Y^{2}}{r}-g_{X Z} \frac{Y Z}{r}+g_{Y Z} \frac{X Z}{r}=0
\end{array}\right.
$$

where $r=\sqrt{X^{2}+Y^{2}+Z^{2}}$ and $\rho=\sqrt{X^{2}+Y^{2}}$. Consider the Taylor expansion of the metric around the unprimed observer world line $(r=0)$, namely

$$
g_{I J}=\eta_{I J}+k_{I J}+h_{I J}+O\left(r^{3}\right)
$$


where $k$ and $h$ are homogeneous polynomials in $X, Y, Z$ of the order 1 and 2 , respectively. Their coefficients can depend on T. We can apply this expansion to (27) and separate equations according to the orders in question. Also, one can easily notice that $k$ and $h$ are odd and even, respectively, under the parity transformation. This allows us to separate those conditions even more. As a result, one obtains the following linear system in which the $k_{I J}$ and $h_{I J}$ are considered as unknown, and $X, Y, Z, \rho$ as given

$$
\left\{\begin{array}{l}
k_{T T}=h_{T T}=0 \\
k_{T X^{i}} X^{i}=0=h_{T X^{i}} X^{i} \\
-k_{T X} X Z-k_{T Y} Y Z+k_{T Z} \rho^{2}=0=-h_{T X} X Z-h_{T Y} Y Z+h_{T Z} \rho^{2} \\
k_{T X} Y-k_{T Y} X=0=h_{T X} Y-h_{T Y} X
\end{array}\right.
$$

$$
\left\{\begin{array}{l}
k_{i j} X^{i} X^{j}=0=h_{i j} X^{i} X^{j} \\
k_{X X} X^{2} Z+2 k_{X Y} X Y Z+k_{Y Y} Y^{2} Z-k_{Z Z} Z \rho^{2}-\left(X^{2}+Y^{2}-Z^{2}\right)\left(k_{X Z} X+k_{Y Z} Y\right)=0 \\
h_{X X} X^{2} Z+2 h_{X Y} X Y Z+h_{Y Y} Y^{2} Z-h_{Z Z} Z \rho^{2}-\left(X^{2}+Y^{2}-Z^{2}\right)\left(h_{X Z} X+h_{Y Z} Y\right)=0 \\
\left(k_{Y Y}-k_{X X}\right) X Y+k_{X Y}\left(X^{2}-Y^{2}\right)-k_{X Z} Z Y+k_{Y Z} X Z=0 \\
\left(h_{Y Y}-h_{X X}\right) X Y+h_{X Y}\left(X^{2}-Y^{2}\right)-h_{X Z} Z Y+h_{Y Z} X Z=0
\end{array}\right.
$$

(28) is a Cramer, homogeneous system of equations so it has only trivial solution. However, (29) admits non-trivial following solution

$$
\left\{\begin{array}{l}
h_{X X}=a Y^{2}+2 b Y Z+c Z^{2} \\
h_{X Y}=-a X Y-b X Z+d Y Z+e Z^{2} \\
h_{X Z}=-b X Y-c X Z-d Y^{2}-e Y Z \\
h_{Y Y}=a X^{2}-2 d X Z+f Z^{2} \\
h_{Y Z}=b X^{2}+d X Y-e X Z-f Y Z \\
h_{Z Z}=c X^{2}+2 e X Y+f Y^{2}
\end{array}\right.
$$

where $a, b, c, d, e, f$ are arbitrary T-dependent functions. The Riemann tensor of this metric necessarily satisfies at $r=0$

$$
R_{\left.T \beta \gamma \delta\right|_{r=0}}=R_{\alpha \beta \gamma \delta} \frac{d p^{\alpha}}{d \tau}=0
$$

as it is stated above. 
One can also find the non-vanishing components of Riemann tensor at $r=0$. Those are

$$
\begin{aligned}
R_{X Y X Y} & =\frac{1}{2}(-a-a-2 a-2 a)=-3 a \\
R_{X Y X Z} & =\frac{1}{2}(-b-b-2 b-2 b)=-3 b \\
R_{X Y Y Z} & =\frac{1}{2}(-2 d-2 d-d-d)=-3 d \\
R_{X Z X Z} & =\frac{1}{2}(-c-c-2 c-2 c)=-3 c \\
R_{X Z Y Z} & =\frac{1}{2}(-e-e-2 e-2 e)=-3 e \\
R_{Y Z Y Z} & =\frac{1}{2}(-f-f-2 f-2 f)=-3 f
\end{aligned}
$$

There are exactly six of them which is the number of independent components of a 3dimensional Riemann tensor. So in general, $R_{\alpha \beta \gamma \delta}$ pulled-back to the surface $T=$ const. can be arbitrary. These conditions are satisfied in particular by any ultrastatic spacetime. From physical point of view, the object of interest is Einstein tensor which takes following form

$$
G_{I J}=R_{I J}-\frac{1}{2} R g_{I J}=-3\left(\begin{array}{cccc}
a+c+f & 0 & 0 & 0 \\
0 & -f & e & -d \\
0 & e & -c & b \\
0 & -d & b & -a
\end{array}\right)
$$

Since all diagonal terms cannot be positive at the same time, matter coupled to gravity in this case needs to be exotic one. Vacuum, on the other hand, implies vanishing of Riemann tensor at the world line of the observer.

\section{DISCUSSION}

Our result can be discussed at several levels. We start from a direct technical interpretation, and proceed toward more specialized ones.

Technically, what we have discovered leads to a relation between spacetimes endowed with the Trautman coordinates: $(g, \tau, r, \theta, \phi)$ and $\left(g^{\prime}, \tau^{\prime}, r^{\prime}, \theta^{\prime}, \phi^{\prime}\right)$ are in the relation provided the map defined by the coordinates via (10) is smooth. We have showed that the map is always differentiable. However, we gave an example of a pair such that the map is not differentiable for the second time. The example was followed by a more general

condition for the pair to be in the relation if $g^{\prime}$ is flat, namely then, a necessary condition is a constraint on the Riemann tensor at the observer's line (10)

$$
\frac{d p^{\mu_{1}}(\tau)}{d \tau} R_{\left.\mu_{1} \mu_{2} \mu_{3} \mu_{4}\right|_{r=0}}=0
$$


where the indices $\mu_{1}, \ldots, \mu_{4}$ refer to any coordinate system non-singular at $r=0$. A general necessary and sufficient condition is not known to us.

From the gauge fixing point of view, the symmetries of adapted coordinates are the residual diffeomorphisms that are not eliminated by our gauge choice. Hence, they are a failler of the corresponding gauge. In the current case of the Trautman coordinates the family of symmetries is for every metric tensor 10 dimensional, however in a general case a symmetry is differentiable only once, and not necessarily twice. Therefore, if one insists on the smoothness of the symmetries, the conclusion is that their family is smaller than 10-dimensional. Hence, the lack of the smoothness we have encountered makes the gauge choice corresponding to the Trautman coordinates actually better, than say the Gauss radial coordinates. An exact dimension of the family of the symmetries is not known at this point. For that we would need a general sufficient and necessary condition for the smootheness of the map (10).

Finally, we can look at the the issue from the point of view of the observer that describes the surrounding spacetime by using the Trautman coordinates. The observer has at her or his disposal the set $M_{\mathrm{obs}}$ of values the quadruples $(\tau, r, \theta, \phi)$ of the coordinates take. The spacetime geometries emerge to the observer in the form of the family of metric tensors that satisfy (8) and the complicated conditions at $r=0$. In $M_{\text {obs }}$ the smooth manifold structure breaks at the points corresponding to $r=0$. The observer may gain insight into that structure by introducing the Cartesian coordinates system (15) corresponding to some metric tensor $g$. According to our results, those coordinates recover correctly the topological and differential (to the first order) structure of spacetime at $r=0$. However, the metric tensor components in those coordinates will in general fail to be twice differentiable. That is indication that the Cartesian coordinates do not encode the spacetime smooth structure. The observer may analyze a structure of the singularity of the second and higher derivatives of $g_{I J}$ and turn it into a set of new laws of physics. In particular she or he will notice, that whenever the coordinates are one more time differentiable at $r=0$, then the Riemann tensor satisfies (26). In a matter of fact, if $g$ is flat in a neighborhood of the line $r=0$, than the smooth structure defined in $M_{\mathrm{obs}}$ by the Cartesian coordinates agrees with that of an actual spacetime. On the other hand, for arbitrary metric tensor there always exists some improved coordinates $\tilde{X}^{\mu}=\tilde{T}, \tilde{X}, \tilde{Y}, \tilde{Z}$ dependent on the metric tensor $g$, non-smooth at $r=0$, making the metric tensor

$$
g_{\mu \nu}(\tilde{T}, \tilde{X}, \tilde{Y}, \tilde{Z}) d \tilde{X}^{\mu} d \tilde{X}^{\nu}
$$

smooth at $r=0$. Those coordinates reflect the actual manifold structure including the points of $r=0$. That structure in observer's space $M_{\mathrm{obs}}$, however, depends on the metric tensor. 


\section{ACKNOWLEDGEMENTS}

This work was partially supported by the Polish National Science Centre grant No. 2011/02/A/ST2/00300.

[1] Trautman A., Radiation and boundary conditions in the theory of gravitation, 1958 Bull. Acad. Polon. Sci., ser. sci. math., astr. et phys. 6 407-412

[2] Bondi H., van der Burg M.G.J and Metzner A.W.K, Gravitational waves in general relativity. 7. Waves from axisymmetric isolated systems, 1962 Proc. Roy. Soc. Lond. A269 21-52

Sachs R.K., Gravitational waves in general relativity. 8. Waves in asymptotically flat spacetimes, 1962 Proc. Roy. Soc. Lond. A270 103-126

[3] Trautman A., Course on General Relativity, 1982 (University of Warsaw)

[4] Duch P. 2012 Dirac observables in general relativity constructed by using the Fermi coordinates Master's thesis under supervision of J. Lewandowski, University of Warsaw

[5] Rovelli C., What Is Observable in Classical and Quantum Gravity?, 1991 Class. Quant. Grav. $8297-316$

Dittrich B., Partial and complete observables for Hamiltonian constrained systems, 2007 Gen. Rel. Grav. 39 1891-1927 arXiv:gr-qc/0411013

Dittrich B., Partial and complete observables for canonical general relativity, 2006 Class. Quant. Grav. 23 6155-6184 arXiv:gr-qc/0507106

Dapor A., Kamiński W., Lewandowski J. and Swieżewski J., Relational Evolution of Observables for Hamiltonian-Constrained Systems, 2013 Phys. Rev. D88 084007 arXiv:1305.0394

[6] Duch P., Kamiński W., Lewandowski J. and Świeżewski J., Observables for general relativity related to geometry, 2014 JHEP 05077 arXiv:1403.8062

Duch P., Kamiński W., Lewandowski J. and Świeżewski J., Addendum: Observables for General Relativity related to geometry, 2015 JHEP 04075 arXiv:1503.07438

Bodendorfer N., Lewandowski J. and Świeżewski J., General relativity in the radial gauge: Reduced phase space and canonical structure, 2015 Phys. Rev. D92 084041arXiv:1506.09164

[7] Bodendorfer N., Duch P., Lewandowski J. and Świeżewski J., The algebra of observables in Gaußian normal spacetime coordinates, 2016 JHEP 01075 arXiv:1510.04154

[8] Heemskerk I., Construction of Bulk Fields with Gauge Redundancy, 2012 JHEP 09106 arXiv:1201.3666

Kabat D., Lifschytz G., Roy S. and Sarkar D., Holographic representation of bulk fields with spin in AdS/CFT, 2012 Phys. Rev. D86 026004arXiv:1204.0126

Kabat D. and Lifschytz G., Decoding the hologram: Scalar fields interacting with gravity, 2014 Phys. Rev. D89 066010 arXiv:1311.3020

[9] Donnelly W. and Giddings S.B., Diffeomorphism-invariant observables and their nonlocal algebra, 2016 Phys. Rev. D93 024030 arXiv:1507.07921

[10] Duch P., Lewandowski J. and Świeżewski J., Observers observables. Residual diffeomorphisms, 2017 Class. Quant. Grav. 34125009 arXiv:1610.03294 